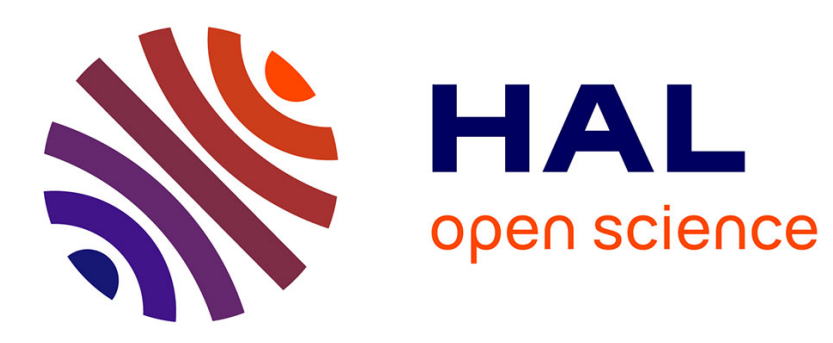

\title{
In Vivo Supervised Analysis of Stent Reendothelialization From Optical Coherence Tomography
}

Claude Kauffmann, Pascal Motreff, Laurent Sarry

\section{- To cite this version:}

Claude Kauffmann, Pascal Motreff, Laurent Sarry. In Vivo Supervised Analysis of Stent Reendothelialization From Optical Coherence Tomography. IEEE Transactions on Medical Imaging, 2010, 29 (3), pp.807-818. 10.1109/TMI.2009.2037755 . hal-02307921

\section{HAL Id: hal-02307921 https://hal.science/hal-02307921}

Submitted on 8 Oct 2019

HAL is a multi-disciplinary open access archive for the deposit and dissemination of scientific research documents, whether they are published or not. The documents may come from teaching and research institutions in France or abroad, or from public or private research centers.
L'archive ouverte pluridisciplinaire HAL, est destinée au dépôt et à la diffusion de documents scientifiques de niveau recherche, publiés ou non, émanant des établissements d'enseignement et de recherche français ou étrangers, des laboratoires publics ou privés. 


\title{
In vivo supervised analysis of stent reendothelialization from optical coherence tomography
}

\author{
Claude Kauffmann ${ }^{1}$, Pascal Motreff ${ }^{2,3}$, and Laurent Sarry ${ }^{2}$ \\ ${ }^{1}$ Department of Medical Imaging, Notre-Dame Hospital, CHUM, Montreal, CANADA \\ ${ }^{2}$ ERIM, Faculty of Medicine, University of Auvergne, Clermont-Ferrand, FRANCE \\ ${ }^{3}$ Department of Cardiology, Gabriel Montpied Hospital, CHU, Clermont-Ferrand, FRANCE
}

\begin{abstract}
The aim of this study is to interactively assess reendothelialization of stents at an accuracy of down to a few microns by analyzing endovascular optical coherence tomography (OCT) sequences. Vessel wall and stent struts are automatically detected by using morphological, gradient and symmetry operators coupled with active contour models; alerts are issued to ask for user supervision over some extreme irregular geometries caused by thrombotic lesions or dissections. A complete distance map is then computed from sparse distances measured between wall and struts. Missing values are interpolated by thin-plate spline (TPS) functions. Accuracy and robustness are increased by taking into account the inhomogeneity of datapoints and integrating in the same framework orthogonalized forward selection of support points, optimal selection of regularization parameters by generalized cross-validation (GCV) and rejection of detection outliers. Validation is performed on simulated data, phantom acquisitions and 11 typical in vivo OCT sequences. The comparison against manual expert measurements demonstrates a bias of the order of OCT resolution (less than 10 microns) and a standard deviation of the order of the strut width (less than 150 microns).
\end{abstract}

Index Terms - Biomedical image processing, coronary artery stenting, optical coherence tomography, neointimal coverage, thin plate spline.

\section{INTRODUCTION}

$\mathrm{C}$ ORONARY endoprosthesis or coronary stents make percutaneous angioplasty safer and help extend the indications. The first generation of stents (BMS, bare-metal stents) in the 1990s marked a major step forward following the development of the antiplatelet treatment protocol. The association of aspirin and thienopyridine during the early months following stent implantation reduced the incidence of acute or subacute thrombosis, ushering in a new era in interventional cardiology. These BMS were nevertheless associated with a risk of restenosis by neointimal hyperplasia observed in the first 6 months after implantation, and giving recurrent angina in 10 to $20 \%$ of cases. Restenosis was even more frequent in diabetic patients, after using more than one stent, in long lesions or in small-diameter coronary arteries.

Over the five last years, drug-eluting stents (DES) have been developed in response to the need to combat high in-stent restenosis rates in these patient subgroups, cutting the rates of recurrent angina and repeat revascularization in the first year by four. However, the drug used against the neointimal hyperplasia also impairs the normal healing processes of the injured arterial wall. The delay in arterial healing and the lack of complete stent coverage by reendothelialization may predispose patients to stent thrombosis. Thus, to avoid a catastrophic event that in the majority of cases will lead to sudden death or myocardial infarction, the dual antiplatelet treatment is continued after the first month. The optimal duration of dual antiplatelet treatment remained unknown.

In clinical practice would benefit greatly if it was possible to observe neointimal coverage after stent implantation: (i) we could analyze the role of delay in arterial healing after a clinical

Copyright (c) 2009 IEEE. Personal use of this material is permitted. However, permission to use this material for any other purposes must be obtained from the IEEE by sending a request to pubs-permissions@ieee.org. event like stent thrombosis, (ii) we could compare different drug-eluting stents and pinpoint the optimal duration of dual antiplatelet treatment, and (iii) we could obtain an individual prognosis before withdrawing antiplatelet treatment because of bleeding or surgery.

To date, neointimal stent coverage after implantation has only been assessed in animal models or using autopsy observations [1],[2].

Optical coherence tomography (OCT) is an intravascular imaging technique that provides the high-image resolution $(<20 \mu \mathrm{m})$ capable of assessing stented arterial segments in vivo. OCT can accurately differentiate the most superficial layers of the arterial wall as well as the stent struts and the vascular tissue surrounding them [3]. OCT could become a reference tool for assessing appropriate healing of stented coronary segments and comparing various types of stents, and could therefore guide optimal antiplatelet therapy to prevent late stent thrombosis.

Recent studies have confirmed the accuracy of OCT for quantifying neointimal hyperplasia and covered-strut rates with different stents [4]-[6]. Current methods of quantification are accurate and offer good inter- and intra-observer reproducibility, but they are still manual and the time-intensive image analysis step remains a major limitation. An automatic algorithm for detecting stent endothelialization from volumetric optical coherence has already been studied [7], but only on an in vitro model. The blood vessel mimic did not reproduce the artifacts and difficulties with human OCT acquisition (move off center or angulation of ImageWire, coronary artery and cardiac movements, suboptimal saline flush) or stent malapposition. A semi-automatic method was developed with good results, but only for quantification of lumen, stent and neointimal areas, without strut coverage assessment [8]. We proposed to develop an automatic and supervised lumen and strut detection algorithm making it possible to calculate prognostic OCT 


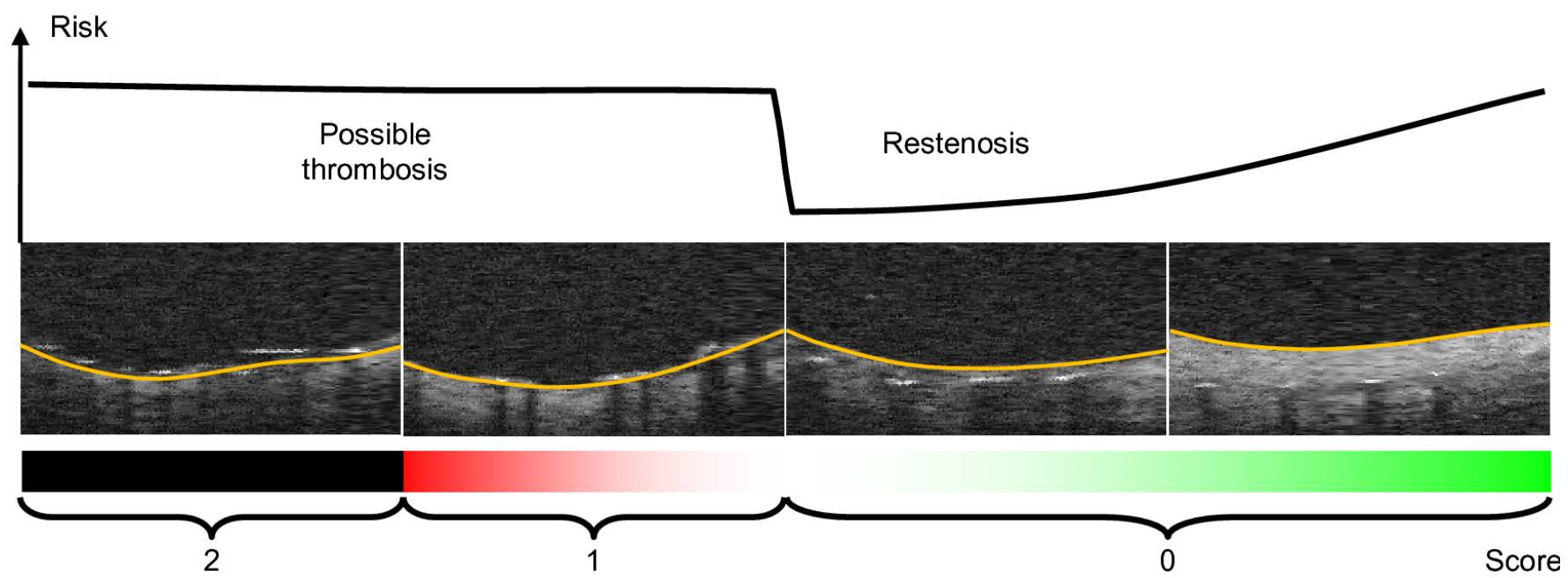

Fig. 1. Schematic view of reendothelialization with the associated thrombosis and restenosis-related risk for the patient. Risk is acute when struts are naked and in direct contact with blood. The orange line shows the actual vessel wall position. Reendothelialization scores and corresponding color scale are given.

parameters used in clinical practice. We compared the algorithm results to conventional measures of neointimal hyperplasia (Fig. 10) and strut coverage on simulated data, phantom data and 11 demonstrative examples of human coronary stent analyzed by OCT 6 months after implantation.

\section{MATERIALS AND METHODS}

\section{A. Optical coherence tomography for endovascular imaging}

\section{1) OCT image formation and acquisition protocol}

OCT is based on low-coherence interferometry. Broad band light sources are used instead of monochromatic laser diodes in order to shorten distance to interference locus to a few micrometers. The optical setup of the system typically consists of a Michelson-type interferometer. The light is split along the two arms of the interferometer: for the reference arm, light is reflected by a reference mirror, whereas for the sample arm, light is reflected by a rotating mirror and backscattered by the tissue of interest. The device actually used for percutaneous coronary interventions (PCI) exploits time-domain OCT (ImageWire M2, LightLab Imaging, Westford, MA, USA), which means that the reference arm is translated axially over time to explore the sample of interest in greater depth and detail. The core of the device is a micro-lens tip fused with a singlemode optical fiber ensuring image transfer. Lateral scanning is done by pullback of the fiber tip into its guide. The image signal is obtained from the amplitude of the interference pattern envelope.

The OCT procedure is generally conducted 6 months after stenting, immediately following the control angiography. $30 \mathrm{UI} / \mathrm{kg}$ non-fractionated heparin is injected intra-arterially by a $6 \mathrm{~F}$ catheter. A 0.014 inch guide is introduced into the vessel and positioned distally to the stent. A HeliosTM coaxial occlusion balloon catheter (OBC), (LightLab Imaging, Westford, MA, USA) is introduced along the guide across the vessel until the balloon marker is at the distal extremity of the stent. The guide is withdrawn and replaced by $1.4 \mathrm{~F}$ optic fiber, connected up to the LightLab (LightLab Imaging, Westford, MA, USA) and inserted through the balloon until distal to the stent. The occlusion balloon is then withdrawn until proximal to the DES and inflated to between 0.4 and $0.7 \mathrm{~atm}$. Physiological saline is then injected downstream of the OBC via its coaxial catheter. $30 \mathrm{~mL}$ is injected during each pullback. OCT gives a clear image once the medium is sufficiently transparent. Automatic light-source pull-back then begins (1 $\mathrm{mm} / \mathrm{s}$, with $15 \mathrm{image} / \mathrm{s}$ acquisition). A $30 \mathrm{~mm}$ DICOM-format video recording is then made of the artery, including the stented segment. The balloon is deflated and the saline injection stopped. Several pull-backs may be required for analyses exceeding $30 \mathrm{~mm}$ in length.

The main advantage of OCT over other invasive imaging techniques such as intravascular ultrasound (IVUS) is resolution: axial and lateral values are decorrelated and equal to 15-20 $\mu \mathrm{m}$ and 25-40 $\mu \mathrm{m}$, respectively, under OCT compared to $100-150 \mu \mathrm{m}$ and 150-300 $\mu \mathrm{m}$ under IVUS. Another key advantage is the size of the imaging core, at $0.4 \mathrm{~mm}$ for OCT and $0.8 \mathrm{~mm}$ for IVUS.

The main disadvantages of OCT are a poor penetration depth in nontransparent tissues; tissue visualization is kept limited to 2-3 $\mathrm{mm}$ in depth for a global scanning area of $7 \mathrm{~mm}$ in diameter. The second severe limitation is the low pullback speed $(1 \mathrm{~mm} / \mathrm{s})$ required by current time-domain OCT devices. Employing balloon occlusion catheters with saline flushes, making the vascular bed transparent, limits visualization to vessel segments shorter than $3.5 \mathrm{~cm}$, as the maximum recommended inflation time is 35 seconds. Fourier-domain OCT devices will soon overcome this limitation by offering tenfold faster image acquisition speeds than existing probes [9].

One practical advantage of the occlusive balloon is that it maintains the probe guide axially with regard to the vessel: therefore the movement of the probe can only be regarded as radial in the slice plane, and does not influence the relative distance between the stent and walls.

2) Image features for stent inspection

The reendothelialization study hinges on detecting two distinct features. 


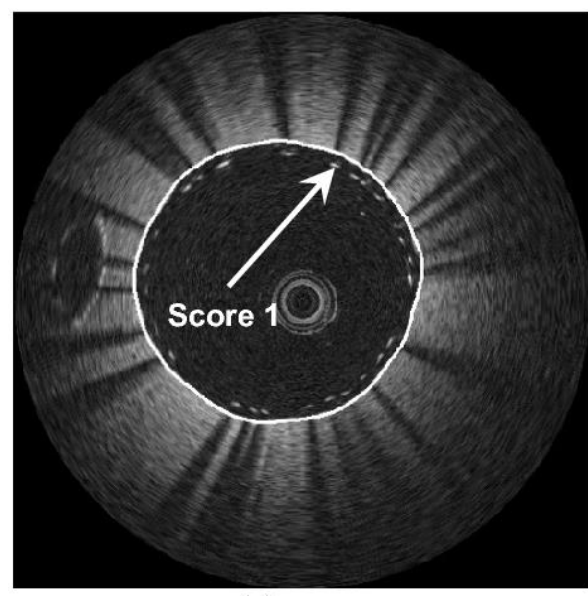

(a)

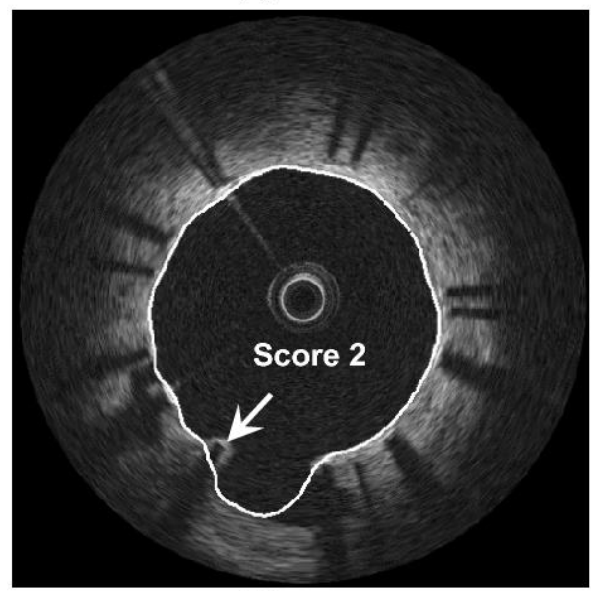

(d)

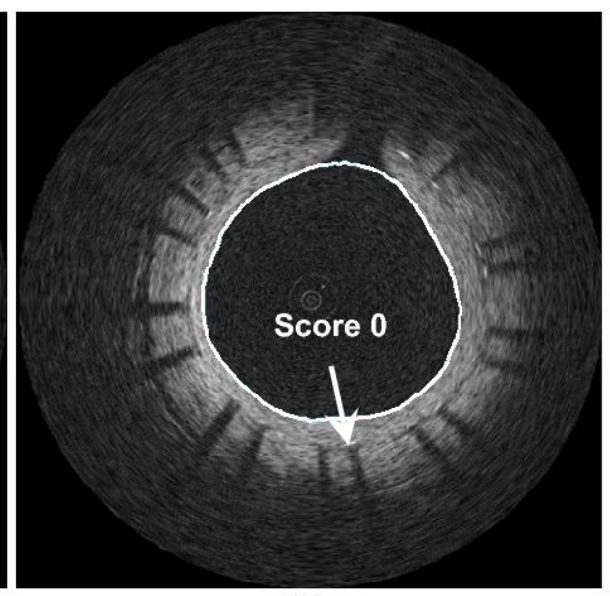

(b)

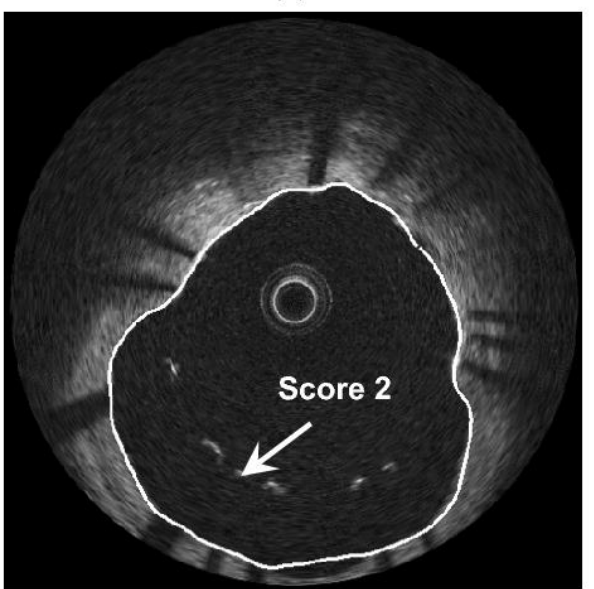

(e)

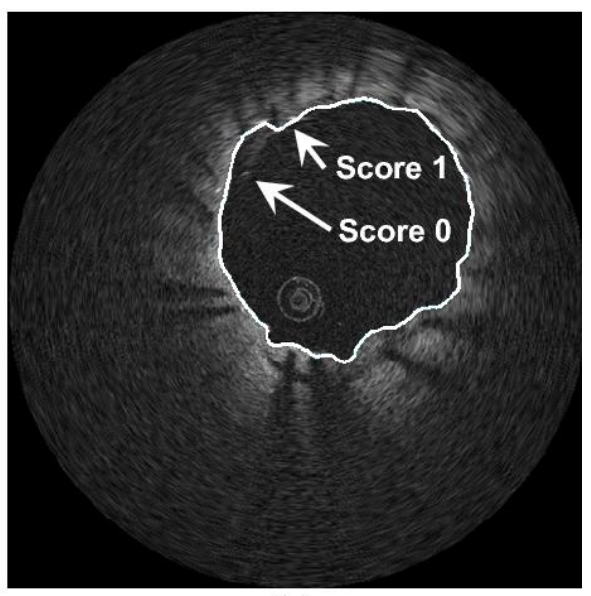

(c)

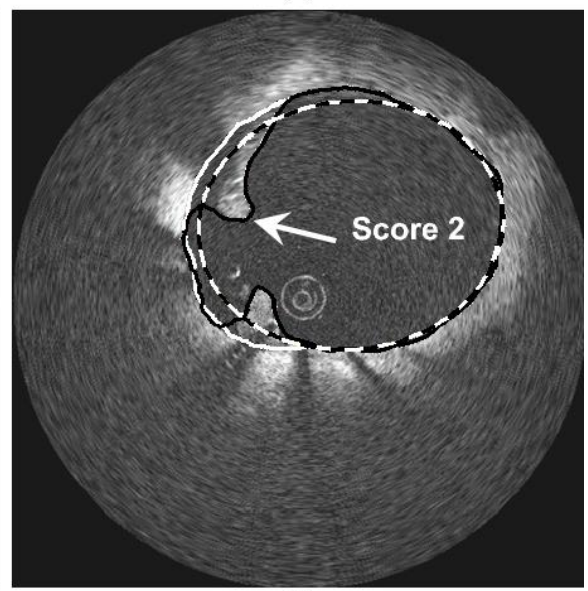

(f)

Fig. 2. Typical images sampled from OCT sequences used for validation. Different configurations of stent position with regard to vessel wall and the corresponding scores are shown (white arrows) together with the results of wall segmentation (white line). Well-apposed stent (top): (a) phantom acquisition simulating uncoverage (the metallic echo is at a distance corresponding to the strut width from the wall); (b) homogeneous and deep coverage; and (c) inhomogeneous coverage. Malapposed stent (bottom): 3 slices of the same OCT sequence at malapposition endings (d) and (f) and middle slice (e). When automatic detection fails to find vessel wall (black line), an ellipse model is fitted to the red contour (dotted line) to detect shape irregularity and an alert is raised for manual correction (white line).

First, the struts that return the metallic echoes of the stent in each slice. The echo location corresponds to the first interface between blood and metal. This means that there is a residual distance corresponding to the width of the wire, even if the stent is fitted well against the vessel wall (Fig. 10(a)). When the wall is contrasted enough, a shadow zone is visible behind every strut. This proves useful for detecting the strut itself, when strut contrast cannot differentiate against the mean speckle level in the wall tissue, which is often the case when reendothelialization happens (Fig. 10(b)). However, this shadow zone is a drawback when the mesh is uncovered or under-deployed, because it hides a part of the vessel wall (Fig. 10(a) and 10(c)) that then needs to be extrapolated somehow. OCT experiences the same geometrical artifacts as IVUS [10]. Indeed, strut echo is magnified as distance to the probe rises. Furthermore, the echo is always oriented orthogonally to the direction of the incident beam, which means that it may not correspond to the orientation of the wall when the probe is offcenter. In two extreme cases, the struts can lie in the vascular bed: firstly, in the event of incomplete apposition, where the distance between echoes and wall is greater than wire width
(Fig. 10(e)); secondly, in the event of branch jail.

Second, the vessel wall boundary, contrast of which depends on distance and orientation in relation to the incident light beam, respectively due to radial attenuation and reflection. Concerning distortions, a vascular segment with a cylindrical shape may yield elliptical cross-sections depending on the orientation of the probe in relation to its axis [10]. Depending on the extent of reendothelialization, the wall edges may be irregular if the struts are not totally covered (Fig. 10(c)), or otherwise smooth (Fig. 10(b)). If reendothelialization keeps growing, vessel lumen area decreases, resulting in restenosis. Figure 10 summarizes the configurations likely to occur, with the exact location of the vessel boundary to be detected and the associated risk for the patient.

Particular difficulty to detect the intimal interface may arise at endings of malaposition (Fig. 10(d) and 10(f)). Indeed, neointimal coverage acts as a 3D process that fills the space between the stent and the wall. It is inexistent in middle slices where the gap is too large (Fig. 10(e)), but may look as a detached layer in intermediate width values (Fig. 10(f)), where user intervention may be required as described below. 


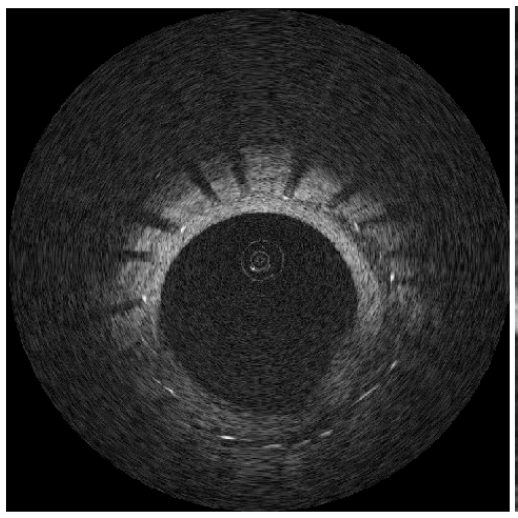

(a)

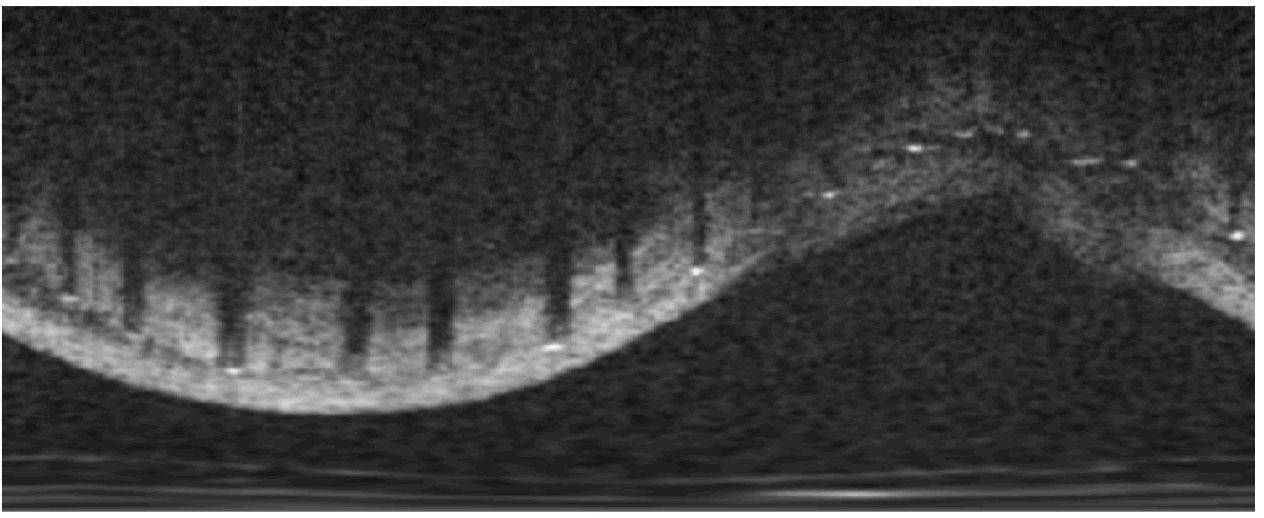

(b)

Fig. 3. (a) typical slice image as delivered by the OCT device, (b) polar reformation of the image in (a); the horizontal and vertical axes represent respectively the angular position between 0 and 360 degrees and the pixel position from image center (probe position) to image border.

\section{B. Image processing for wall and strut detection}

\section{1) Processing overview}

Due to the characteristics of the wall and struts previously described, image segmentation is made of three independent processes:

- the first part deals with the delineation of vessel bed edges. Depending on the amount of reendothelialization, those edges correspond to the interface of blood with metal and/or with endothelium.

- in case of strong malapposition stemming from the presence of thrombus or wall dissection, the exact wall boundary is sometimes difficult to recover. Fortunately, the detected border is almost always irregular and an alert can be automatically sent to the user to control the detection result and correct it if necessary.

- the last part deals with the strut segmentation. The radial shadow zones behind the struts are searched to infer whether a strut is present. Moreover, when the shadow zones are missing, the strut intensity is itself also

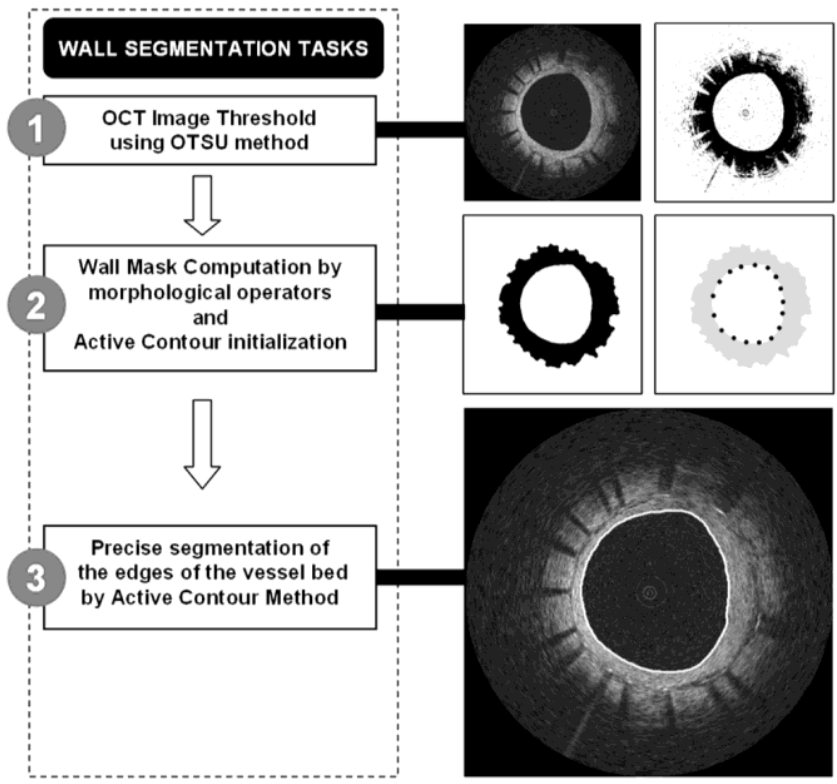

Fig. 4. Steps involved in the vessel bed edge segmentation phase. Step 1 is performed once for the 3D OCT image set. Steps 2 and 3 are applied successively in a slice-by-slice process. considered for strut detection and localization.

2) Wall detection

Vessel bed edge segmentation is obtained in a three-step process as illustrated in Fig. 10. At step 1, an automatic threshold level is computed on the gray-scale histogram of the 3D OCT image set using the Otsu method [12]. This threshold level is then applied to all cross-sectional OCT images in order to obtain a binary OCT image set. This binary image set is used at step 2 to find a rough approximation of the wall border. For this, each OCT section is run through a morphological segmentation process as presented in [11]:

$$
M_{i}=\left(H\left(T_{i} \circ K_{1}\right)\right) \cdot K_{2} \text {, }
$$

where $T_{i}$ is the $i^{\text {th }}$ thresholded OCT slice image, $H$ is a holefilling function, and $K_{1}, K_{2}$ are the structuring elements used by the function processing morphological opening and closing, respectively. The structuring elements $K_{1}, K_{2}$ are binary discs with a given radius of $r_{1}=2$ and $r_{2}=9$ respectively. The size of $K_{1}$ and $K_{2}$ was defined empirically, and the same value was used for the segmentation of all OCT image sequences. As shown in Fig. 10, the resulting morphological image $M_{i}$ is a clean binary image of the vessel wall, where noise speckle, struts and artifacts (such as the guide at the image center) have been removed. The wall border can be sampled by profile analysis from lines drawn from the image center to the border at different angular positions. The detected wall position, represented by black points on Fig. 10, is used to initialize an active contour near to the real edges of the vessel bed. At step 3 , the vessel bed edges are precisely segmented via a segmentation process based on the active contour model described in [11].

This three-step automatic wall segmentation process was applied to all four OCT sequences and performed very well, regardless of the changing image quality, as shown on Fig. 10. Moreover, the active contour segmentation approach implicitly interpolates the local wall boundary gaps due to vessel branching, as well as the wall signal inhomogeneity shadow zone produced by struts localized between the OCT sources and the wall boundaries.

\section{3) Alert on wall irregularity}

In order to cut the time needed to run the qualitative clinical validation of the vessel wall segmentation results, we perform an automatic shape analysis of the vessel wall contours on every 


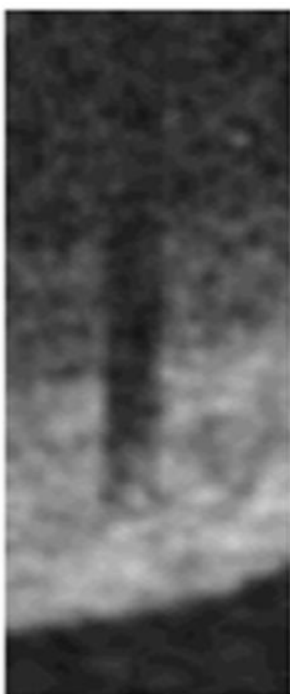

(a)

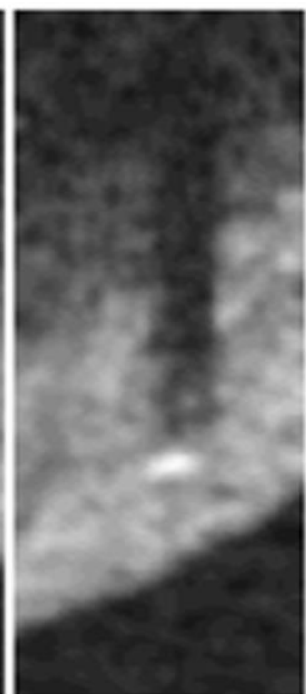

(b)

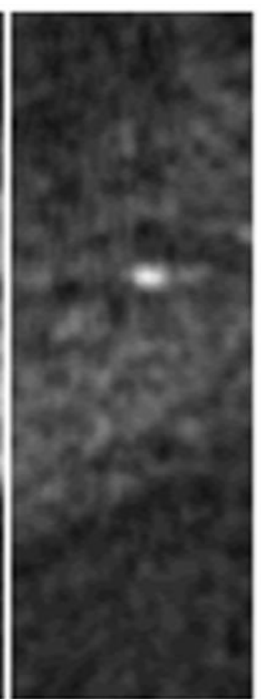

(c)
Fig. 5. Typical strut appearances in OCT acquisition: (a) a shadow zone is visible but no strut echo; (b) both shadow zone and strut echo are visible; and (c) a strut echo is visible but no shadow zone.

OCT slice. As the vessel contour is normally expected to be circular or elliptic, an ellipse is fitted to the segmented vessel wall contours on each OCT image [13]. The discrepancy between the vessel wall contour and the fitted ellipse is computed and used to trigger an alert for user validation if required.

The difference between the segmented vessel contour and the ellipse model is computed as the Mean Square Error (MSE), as follows:

$$
e=\sqrt{\frac{1}{n} \sum_{i=1}^{n}\left(d_{i}^{\perp}\right)^{2}},
$$

where $d_{i}^{\perp}$ is the orthogonal distance from the ith point of the contour to the fitted ellipse. A simple alert threshold $e>T_{A}$ was used to inform the user on which contours should be qualitatively validated and corrected if needed. Figures 10(d) and (f) give typical vessel wall shapes that trigger an alert.

\section{4) Strut detection}

The strut segmentation process presented in this section is performed in a slice-by-slice process. In order to simplify the strut segmentation algorithms, each OCT slice image is reformatted and interpolated in polar coordinates as shown in Fig. 10. Each point $P(x, y)$ in image (a) is transformed to a corresponding point $P(\rho, \theta)$ in image (b), where $\rho$ represents the radial Euclidian distance from the pole, which is the center of image (a), and the $\theta$ coordinate represents the counterclockwise angle from the $0^{\circ}$ ray known as the positive $\mathrm{X}$-axis.

A strut in an OCT slice image does not have a unique and constant appearance. In the best case, it appears as a highcontrast speckle of variable length (from one to 15 pixels) depending on the orientation of the OCT probe in relation to the stent. Figure 10 shows three typical appearances of a strut in a given OCT acquisition image (zoomed parts of the image on Fig. 10(b)). These three examples show that it is not simple to detect all struts, and that different characteristics must be taken in account to perform robust and precise detection. In cases (a) and (b), we show that as the contrast of the vessel wall is high, the strut produces a radial shadow zone that can be efficiently used to infer whether a strut is present. Figure 10 illustrates the method used to infer the presence of a strut based on the detection of the shadow zones. First, as shown on Fig. 10 (middle image), the $\mathrm{x}$-axis gradient component $\mathrm{g}_{\mathrm{x}}$ of the polar OCT slice image (top image) was computed in order to enhance the vertical lines produced by the borders of the shadow zone. Secondly, we compute the cumulative of $g_{x}$ along the y-axis for each position $x$. The resulting curve illustrated in Fig. 10 (bottom) is screened for all specific increasing monotonic signal transitions, starting at a local minima below zero and ending at a local maxima greater than zero. Fig. 10 illustrates a typical signature of signal transition in presence of a shadow zone. We define two metrics to detect a shadow zone. The first is the peak-to-peak amplitude of the transition signal $f_{a}$ in (3), and the second represents the symmetry between the absolute values of $L_{\text {max }}$ and $L_{\text {min }}$, noted $f_{b}$ in (4):

$$
\begin{gathered}
f_{a}(i)=L_{\text {max }}(i)-L_{\text {min }}(i), \\
f_{b}(i)=L_{\text {max }}(i)-\left|L_{\text {min }}(i)\right|,
\end{gathered}
$$

where $i$ stands for the $i^{\text {th }}$ signal transition, $L_{\text {max }}(i)$ always $>0$ and $L_{\min }(i)$ always $<0$.

A normalized metric of symmetry, $S(i)$ based on $f_{a}(i)$ and $f_{b}(i)$ is used in (5), where $S(i)=1$ represents perfect symmetry and $S(i)=0$ is perfect asymmetry:

$$
S(i)=1-f_{b}(i) / f_{a}(i)
$$

A shadow zone is then detected as $f_{a}(i)>T_{1}$ and $S(i)>T_{2}$,

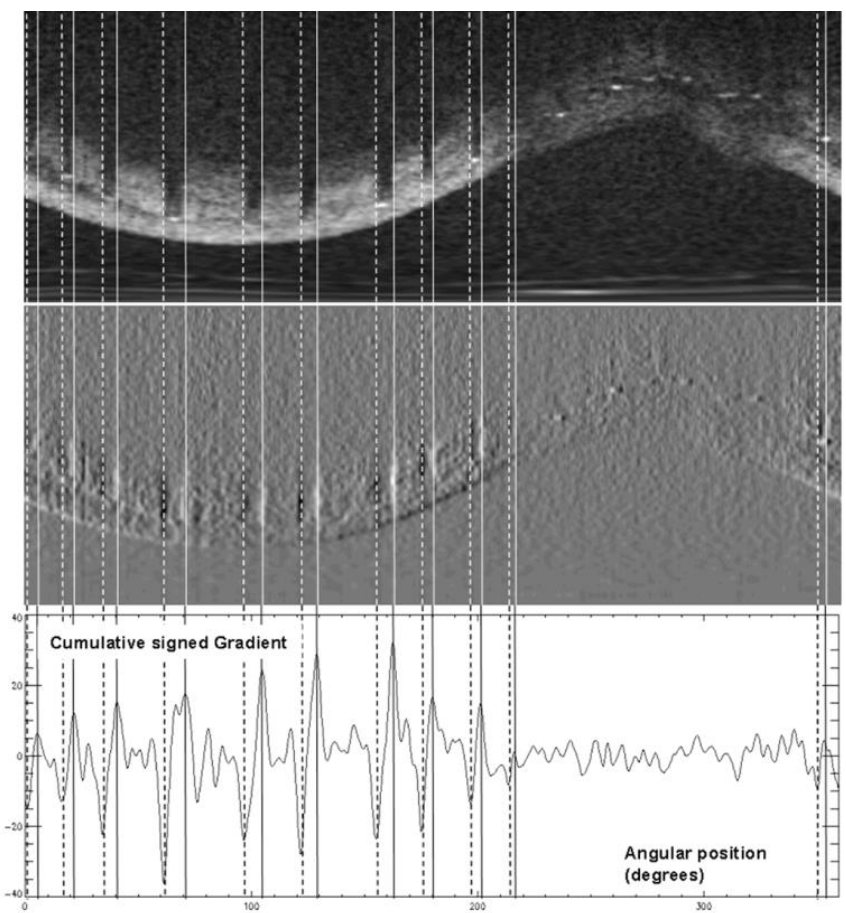

Fig. 6. Automatic detection of radial shadow zones behind the struts. At the top, we show the polar-reformatted OCT slice image of Fig. 10(b). The middle and bottom images represent the signed enhanced gradient along the $\mathrm{x}$-axis and the cumulative signed gradient along the $y$-axis, respectively. Vertical lines represent the shadow zone border detection, where the left borders correspond to local minima of the curve (dotted line) and the right borders correspond to local maxima of the curve (continuous line). 


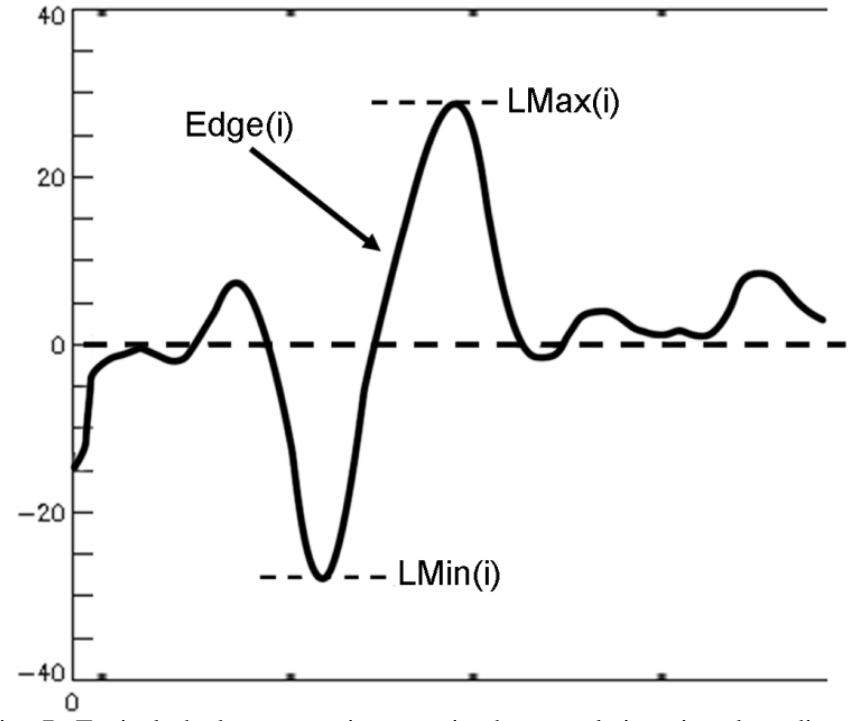

Fig. 7. Typical shadow zone signature in the cumulative signed gradient image.

where $T_{1}$ and $T_{2}$ are threshold values set empirically with regard to the values of $f_{a}(i)$ and $S(i)$ generated by noise and artifacts, when no shadow zone exists.

The final step is to detect the exact strut position. When a shadow zone is detected (Fig. 10(a) and 10(b)), we analyze gray level radial profiles sampled every quarter degree. A strong decrease in gray level should be present just after the strut when moving from the lumen wall to the image border. When strut signal is not visible (Fig. 10(a)), the strut is considered to lie at the beginning of the decreasing part. Multiple occurrences for several adjacent profiles inside the shadow zone are checked for, because the larger the detected features are, the higher the corresponding strut presence probability is, as narrow echoes are most likely to be artifacts..

In the case illustrated in Fig. 10(c), the strut appears without shadow zone because the vessel wall is not highlighted. Strong echoes with a characteristic gray level peak over 200 (on an 8bit scale) are generally characteristic for a strut and are sought for inside the vascular wall. Again detection consistency depends on strut length and probability selection threshold is even set higher than previously as artifacts are more likely to occur in the vessel wall due to speckle noise.

Detection thresholds are initialized from the detection of the shadow zones. They are tuned in order to minimize false positive detections (outliers): false negative detections are naturally handled by the following interpolation scheme, and also outliers but only if they are minority.

\section{Robust interpolation of a distance map and implementation of a score map}

At this stage, the distance $d_{i}$ to the vessel wall is only known at locations $\left(\theta_{i}, z_{i}\right)$ of the $p$ detected struts. Due to detection uncertainties, some of the struts are missed, while others are outliers, i.e. their locations are totally abnormal. These outliers are really problematic because they can be falsely interpreted as under-deployed struts. Thus, it has to be possible to interpolate missing information and reject outliers at the same time.

In order to achieve isotropic sampling in the $(\theta, z)$ plane, axis $\theta$ is scaled so that one unit corresponds to a circular arc equal to one unit in direction $z$.

\section{1) Approximation model}

A thin-plate spline (TPS) model was chosen in order to simulate the rigidity of the stent. Contrary to tensor product representations such as B-splines, TPS is adapted to datapoints that are not regularly sampled over a grid. Strut density depends on the mesh drawing and on the location of the probe inside the vessel lumen. Any tortuous vessel is likely to lie against the wall, and the further the struts are, the closer they will be from each other in the $(\theta, z)$ plane.

TPS function $f$ is defined so as to minimize the sum of squared errors to the data ESSE:

$$
E_{S S E}=\sum_{i=1}^{p}\left(f\left(\theta_{i}, z_{i}\right)-d_{i}\right)^{2}
$$

and to the bending energy $E_{f}$ :

$$
E_{f}=\iint_{\mathbb{R}^{2}}\left(f_{\theta \theta}^{2}-2 f_{\theta z}^{2}+f_{z z}^{2}\right) d \theta d z
$$

The solution [16] is made up of an affine part representing the behavior of $f$ at infinity and a sum of the function $U(r)=$ $r^{2} \log (r)$, bounded and asymptotically flat, centered at the struts $\left(\theta_{i}, z_{i}\right)$ :

$$
\begin{aligned}
& f(\theta, z)=a_{1}+a_{\theta} \theta+a_{z} z \\
& +\sum_{i=1}^{p} w_{i} U\left(\left\|\left(\theta_{i}, z_{i}\right)-(\theta, z)\right\|\right),
\end{aligned}
$$

where TPS weights $w_{i}$ are subject to: $\sum_{i=1}^{p} w_{i}=\sum_{i=1}^{p} w_{i} x_{i}=\sum_{i=1}^{p} w_{i} y_{i}=0 . \quad$ These conditions, combined with the knowledge of the real-valued distance $d_{i}$ at locations $\left(\theta_{i}, z_{i}\right)$, give the following linear system:

$$
\mathbf{H} \mathbf{w}^{\prime}=\mathbf{H}\left[\begin{array}{l}
\mathbf{a} \\
\mathbf{w}
\end{array}\right]=\left[\begin{array}{ll}
\mathbf{O} & \mathbf{P}^{\mathrm{T}} \\
\mathbf{P} & \mathbf{K}
\end{array}\right]\left[\begin{array}{l}
\mathbf{a} \\
\mathbf{w}
\end{array}\right]=\left[\begin{array}{l}
\mathbf{0} \\
\mathbf{d}
\end{array}\right]=\mathbf{y},
$$

where $K_{i j}=U\left(\left\|\left(\theta_{i}, z_{i}\right)-(\theta, z)\right\|\right)$, the $i^{\text {th }}$ row of $P$ is $\left(1 x_{i} y_{i}\right), \mathbf{0}$ is a $3 \times 3$ null matrix, $\mathbf{o}$ is a $3 \times 1$ null column vector, $\mathbf{w}$ and $\mathbf{d}$ are column vectors formed from $w_{i}$ and $d_{i}$, respectively, and $\mathbf{a}$ is the column vector containing elements $a_{1}, a_{\theta}$ and $a_{z}$.

The presence of noise and outliers makes it necessary to use approximation instead of exact interpolation. This is done by minimizing $E_{S S E}+\lambda E_{f}$ (6-7), which equates to replacing $\mathbf{K}$ by $\mathbf{K}_{\lambda}$ in (9):

$$
\mathbf{K}_{\lambda}=\mathbf{K}+\lambda \mathbf{I}_{p},
$$

where $\lambda$ is a regularization parameter that controls the bending of the TPS. Criteria used for its choice are detailed in paragraph 4 below.

\section{2) Optimal weights for spatial density compensation and distance adaptivity}

Due to heterogeneities in vessel wall contrast, distance information is liable to be lacking in some regions of the $(\theta, z)$ plane where struts are not detected. Therefore, a specific weighting process has been designed to take into account the spatial density of datapoints. A Voronoi diagram is built from all $\left(\theta_{i}, z_{i}\right)$ locations. It is decimated so that every cell has a significant surface higher than the squared dimensions of one strut. To do so, the smallest cells are iteratively deleted until the 


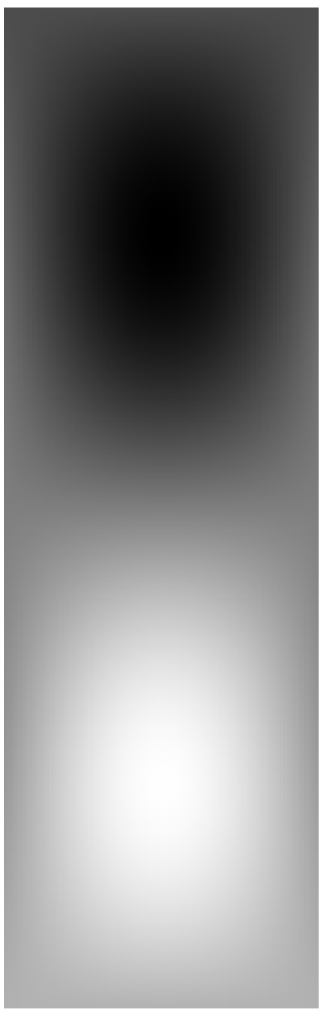

(a)

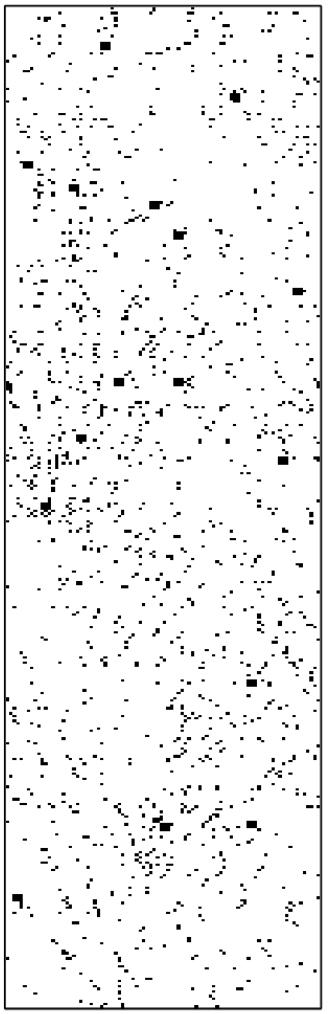

(b)

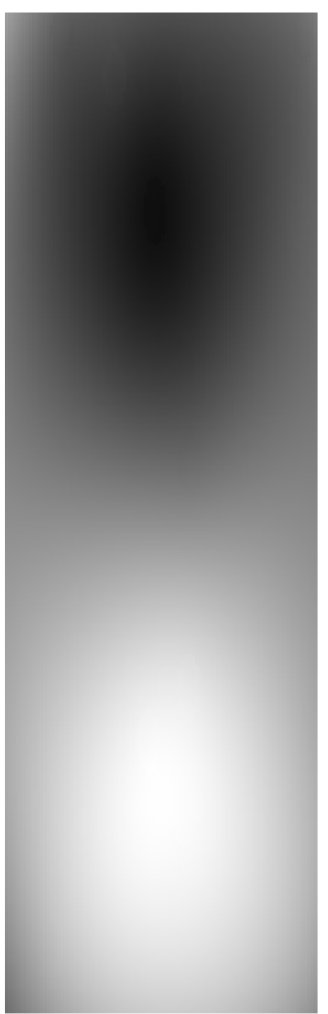

(c)

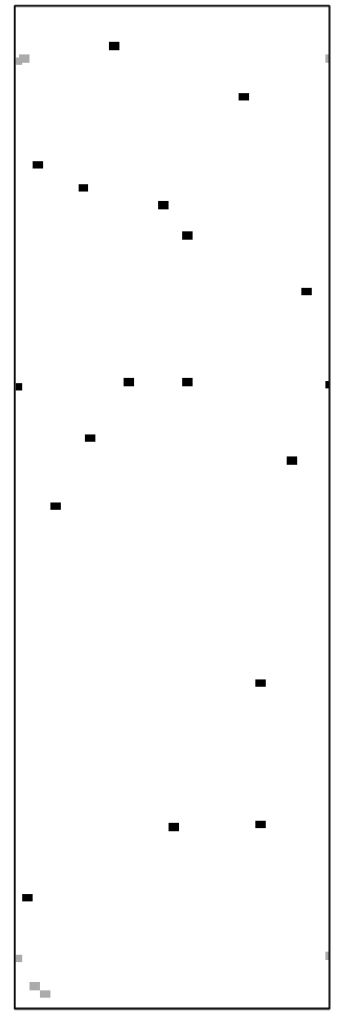

(d)

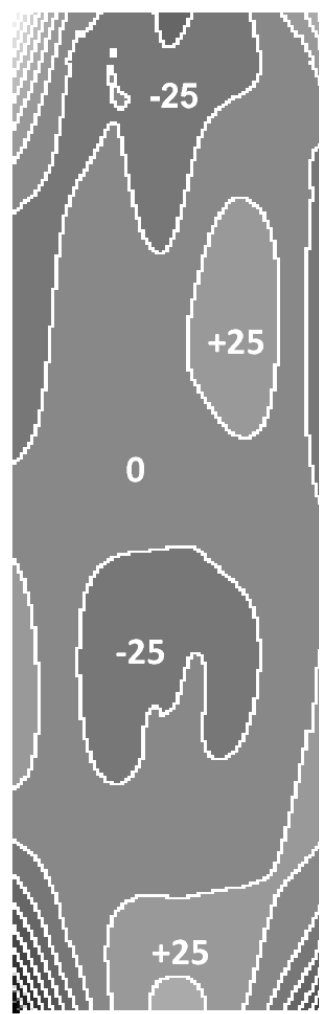

(e)

Fig. 8. Results of depth map interpolation on simulated data corresponding to conditions \#10 in Tab. 0: (a) ground truth map, (b) locations of struts used for interpolation and outliers (squares), (c) estimated map, (d) locations of estimated outliers (true in black and false in gray) and (e) difference with ground truth.

minimal acceptable size is reached. The distance for the current deleted cell is divided between the neighboring cells with a ratio depending on the gain in area after deletion. Areas $s_{i}$ of polygon cells are used to compute final weights $\mu_{i}(11)$.

The approximation must also take into account the fact that accuracy must be higher for small distances because they are associated with a much higher risk for the patient. Therefore, a monotonically decreasing function is applied to distance:

$$
\mu_{i}=\frac{s_{i}}{1+\left\lfloor d_{i}\right\rfloor}
$$

$\mu_{i}$ values are used to weight squared errors in (7). The linear system (9) is only modified by dividing regularization parameter $\lambda$ located on the diagonal by weights $\mu_{i}$. Density compensation and distance adaptivity are abbreviated as DC and DA respectively in the following.

\section{3) Orthogonalized forward selection of support points}

Linear system contains the full design matrix $\mathbf{K}, \mathbf{K}$ i.e. so that all datapoints serve as support vector for the TPS model. However, the solution for TPS coefficients is in $O\left(p^{3}\right)$, which is too prohibitive when $p$ gets large. A workaround solution consists in selecting a subset of $m$ samples from the initial data, and the full design matrix $\mathbf{H}$ becomes $\mathbf{H}_{\mathrm{m}}$, i.e. a $(p+3) \times$ $(m+3)$ matrix where the last $m$ columns of which correspond to the m support points [14].

Methods inspired from machine learning such as forward selection [15] can be employed to select the centers of the radial basis functions. This consists in recursively adding the most significant centers to the affine terms. For simplification, the affine and radial basis parts are estimated separately: first the coefficients for the affine part are estimated in a least-square sense:

$$
\mathbf{a}=\left(\mathbf{P}^{\mathrm{T}} \mathbf{P}\right)^{-1} \mathbf{P}^{\mathrm{T}} \mathbf{d} .
$$

Then, the estimation for the radial function weights is performed on the residuals $\mathbf{r}=\mathbf{d}-\mathbf{P a}$. This is equivalent to ridge regression [17], i.e. minimization of first order regularization energy $E_{r}$ equal to SSE plus $\lambda$ times $\mathbf{w}_{m}^{\mathrm{T}} \mathbf{w}_{m}$; the weights $\mathbf{w}_{\mathrm{m}}$ associated to the $m$ support points are given by:

$$
\mathbf{w}_{m}=\left(\mathbf{H}_{m}^{\mathrm{T}} \mathbf{H}_{m}+\lambda \mathbf{H}_{m}\right)^{-1} \mathbf{H}_{m}^{\mathrm{T}} \mathbf{r} .
$$

The choice of the $m$ best centers represents a computational burden unless forward selection is used. This consists in picking the centers one after the other from among the remaining set until the decrease in a model selection criterion (MSC) is sufficiently low. At iteration $m$, the design matrix $\mathbf{H}_{m-1}$ is augmented by $\mathbf{h}_{m}$, the column of the full design matrix corresponding to the point that minimizes $E_{r}$.

It has been proven by Chen et al. [5] that Gram-Schmidt orthogonalization of the design matrix $\mathbf{H}_{m}$ in $\widetilde{\mathbf{H}}_{m}$, so that $\mathbf{H}_{m}=$ $\widetilde{\mathbf{H}}_{m} \mathbf{U}_{\mathbf{m}}$ with $\mathbf{U}_{m}$ an upper triangular, is likely to significantly speed up forward selection. Instead of choosing $\mathbf{h}_{\mathrm{m}}$ among the columns of $\mathbf{H}$, its regularized version $\tilde{\mathbf{h}}_{m}$ is chosen from $\widetilde{\mathbf{H}}_{m}$ iteratively updated in the following way:

$$
\widetilde{\mathbf{H}}_{m}=\widetilde{\mathbf{H}}_{m-1}-\frac{\tilde{\mathbf{h}}_{m} \tilde{\mathbf{h}}_{m}^{\mathrm{T}} \widetilde{\mathbf{H}}_{m-1}}{\tilde{\mathbf{h}}_{m}^{\mathrm{T}} \tilde{\mathbf{h}}_{m}} \text { with } \widetilde{\mathbf{H}}_{0}=\mathbf{H}
$$

and minimization of $E_{r}$ means that $\tilde{\mathbf{h}}_{j}$ must maximize the gap in energy between two selections:

$$
\Delta E_{r m}(j)=\frac{\left(\mathbf{r}^{\mathrm{T}} \tilde{\mathbf{h}}_{j}\right)^{2}}{\lambda+\tilde{\mathbf{h}}_{j}^{\mathrm{T}} \tilde{\mathbf{h}}_{j}} .
$$

The transition matrix $\mathbf{U}_{m}$ itself is updated by: 


$$
\mathbf{U}_{m}=\left[\begin{array}{cc}
\mathbf{U}_{m-1} & \left(\widetilde{\mathbf{H}}_{m-1}^{\mathrm{T}} \widetilde{\mathbf{H}}_{m-1}\right)^{-1} \widetilde{\mathbf{H}}_{m-1}^{\mathrm{T}} \mathbf{h}_{m} \\
\mathbf{0}_{m-1}^{\mathrm{T}} & 1
\end{array}\right],
$$

with $\widetilde{\mathbf{U}}_{0}=1$ and makes it possible to recover final weights:

$$
\mathbf{w}_{m}=\mathbf{U}_{m}^{-1} \widetilde{\mathbf{w}}_{m}
$$

from regularized weights:

$$
\widetilde{\mathbf{w}}_{m}=\frac{\mathbf{r}^{\mathrm{T}} \tilde{\mathbf{h}}_{m}}{\lambda+\tilde{\mathbf{h}}_{m}^{\mathrm{T}} \tilde{\mathbf{h}}_{m}},
$$

simply by back-substitution.

\section{4) Optimal regularization parameter}

The MSC used as stopping criterion for the previous iterative algorithm is generalized cross-validation (GCV) [18],[19] as it is considered to avoid data overfit. In [17], it is expressed as:

$$
\begin{aligned}
& G C V_{m}=\frac{\mathbf{r}^{\mathrm{T}} \mathbf{P}_{m}^{2} \mathbf{r}}{\left(\operatorname{trace}\left(\mathbf{P}_{m}\right)\right)^{2}} \\
& =\frac{p}{\left(p-\gamma_{m}\right)}\left(\mathbf{r}^{\mathrm{T}} \mathbf{r}\right. \\
& -\sum_{\mathbf{j}=1}^{m} \frac{\left(2 \lambda+\tilde{\mathbf{h}}_{j}^{\mathrm{T}} \tilde{\mathbf{h}}_{j}\right)\left(\mathbf{r}^{\mathrm{T}} \tilde{\mathbf{h}}_{j}\right)^{2}}{\left.\left(\lambda+\tilde{\mathbf{h}}_{j}^{\mathrm{T}} \tilde{\mathbf{h}}_{j}\right)^{2}\right),}
\end{aligned}
$$

where $\mathbf{P}_{m}=\mathbf{I}_{p}-\widetilde{\mathbf{H}}_{m}\left(\widetilde{\mathbf{H}}_{m}^{\mathrm{T}} \widetilde{\mathbf{H}}_{m}+\lambda \widetilde{\mathbf{H}}_{m}\right)^{-1} \widetilde{\mathbf{H}}_{m}^{\mathrm{T}}$ is the matrix projecting the data $\mathbf{r}$ perpendicular to the space spanned by the model.

Orr [17] has shown that the estimate of regularization parameter $\lambda$ can be inserted in the algorithm, and that its value can be refined at every iteration just after selection of the new center, by means of a re-estimation formula coming from the minimization of $\mathrm{GCV}_{\mathrm{m}}$ and the annulation of its derivative:

$$
\lambda=\frac{\left(\partial \operatorname{trace}\left(\mathbf{P}_{m}\right) / \partial \lambda\right)\left(\mathbf{r}^{T} \mathbf{P}_{m}^{2} \mathbf{r}\right)}{\left(\operatorname{trace}\left(\mathbf{P}_{m}\right)\right)^{2} \widetilde{\mathbf{w}}_{m}^{\mathrm{T}}\left(\widetilde{\mathbf{H}}_{m}^{\mathrm{T}} \widetilde{\mathbf{H}}_{m}+\lambda \widetilde{\mathbf{H}}_{m}\right)^{-1} \widetilde{\mathbf{w}}_{m}} .
$$

The practical computation of (19) and (20) requires the following relationships:

$$
\begin{gathered}
\frac{\partial \operatorname{trace}\left(\mathbf{P}_{m}\right)}{\partial \lambda}=\sum_{\mathbf{j}=1}^{m} \frac{\tilde{\mathbf{h}}_{j}^{\mathrm{T}} \tilde{\mathbf{h}}_{j}}{\left(\lambda+\tilde{\mathbf{h}}_{j}^{\mathrm{T}} \tilde{\mathbf{h}}_{j}\right)^{2}}, \\
\mathbf{r}^{\mathrm{T}} \mathbf{P}_{m}^{2} \mathbf{r}=\mathbf{r}^{\mathrm{T}} \mathbf{r}-\sum_{\mathbf{j}=1}^{m} \frac{\left(2 \lambda+\tilde{\mathbf{h}}_{j}^{\mathrm{T}} \tilde{\mathbf{h}}_{j}\right)\left(\mathbf{r}^{\mathrm{T}} \tilde{\mathbf{h}}_{j}\right)^{2}}{\left(\lambda+\tilde{\mathbf{h}}_{j}^{\mathrm{T}} \tilde{\mathbf{h}}_{j}\right)^{2}}, \\
\operatorname{trace}\left(\mathbf{P}_{m}\right)=p-\sum_{\mathbf{j}=1}^{m} \frac{\tilde{\mathbf{h}}_{j}^{\mathrm{T}} \tilde{\mathbf{h}}_{j}}{\lambda+\tilde{\mathbf{h}}_{j}^{\mathrm{T}} \tilde{\mathbf{h}}_{j}^{\prime}} \\
\text { and } \widetilde{\mathbf{w}}_{m}^{\mathrm{T}}\left(\widetilde{\mathbf{H}}_{m}^{\mathrm{T}} \widetilde{\mathbf{H}}_{m}+\lambda \widetilde{\mathbf{H}}_{m}\right)^{-1} \widetilde{\mathbf{w}}_{m}=\sum_{\mathbf{j}=1}^{m} \frac{\left(\mathbf{r}^{\mathrm{T}} \tilde{\mathbf{h}}_{j}\right)^{2}}{\left(\lambda+\tilde{\mathbf{h}}_{j}^{\mathrm{T}} \tilde{\mathbf{h}}_{j}\right)^{3}} .
\end{gathered}
$$

\section{5) Robust regression and outlier rejection}

Both forward selection and GCV estimation are very sensitive to the presence of outliers in the data. Therefore, we have introduced an M-estimation stage [20] into the loop at iteration $m$, just after center selection, in order to compute a robust value for weight $\widetilde{\mathbf{w}}_{m}$. An iterative reweighted leastsquare approach is used, starting from the initial residuals at iteration $m$ :

$$
\mathbf{r}_{m}^{(0)}=\mathbf{r}_{m-1}=\mathbf{r}-\widetilde{\mathbf{H}}_{m-1} \widetilde{\mathbf{w}}_{m-1},
$$

and the initial value $\widetilde{\mathbf{w}}_{m}^{(0)}$ for the weight comes from the ordinary least square solution (18).

The following iterations are performed until the change in weight value is not significant:

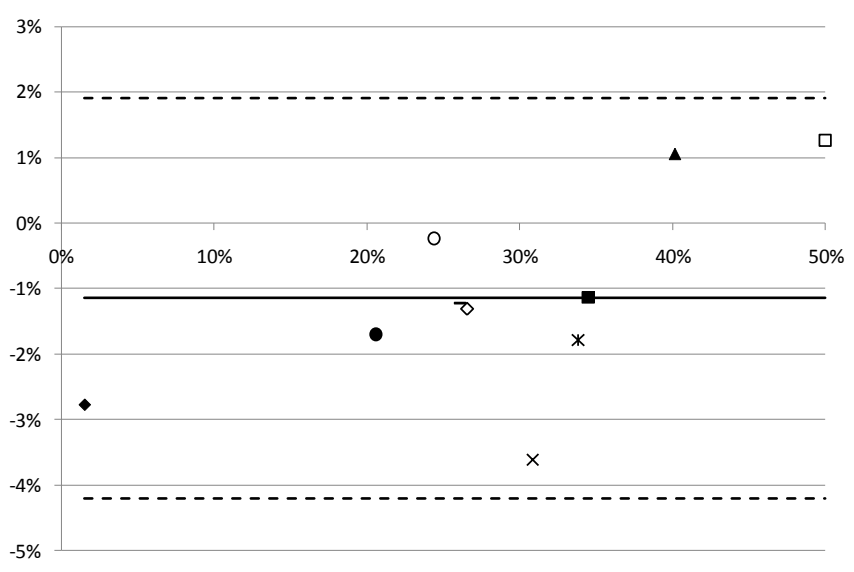

(a)

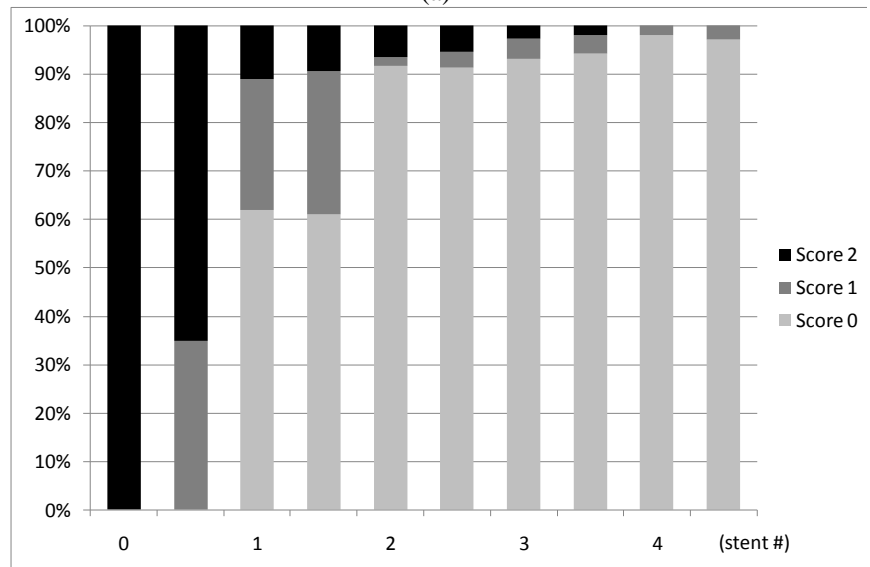

(b)

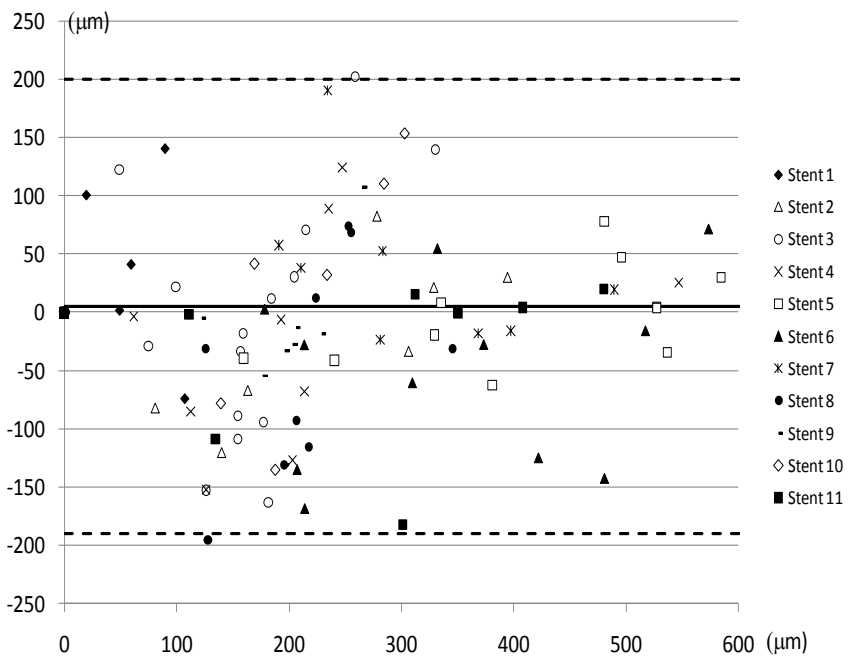

(c)

Fig. 9. Quantitative and qualitative comparisons of the supervised method versus gold standard (expected values for phantom or manual measurement for in vivo acquisitions): (a) Bland and Altman plot of the reendothelialization ratio in \%. The bias mean is $-1.14 \%$ and the $95 \%$ confidence interval is equal to ]-4.20;1.91[; (b) score bar chart for neointimal coverage. Only patients with coverage defect (scores 1 or 2) are presented; and (c) Bland and Altman plot of reendothelialization width in $\mu \mathrm{m}$. The bias mean is $5.3 \mu \mathrm{m}$ and the $95 \%$ confidence interval is equal to ]-189.9;200.5[. For the sake of clarity, one point out of 25 is drawn. 


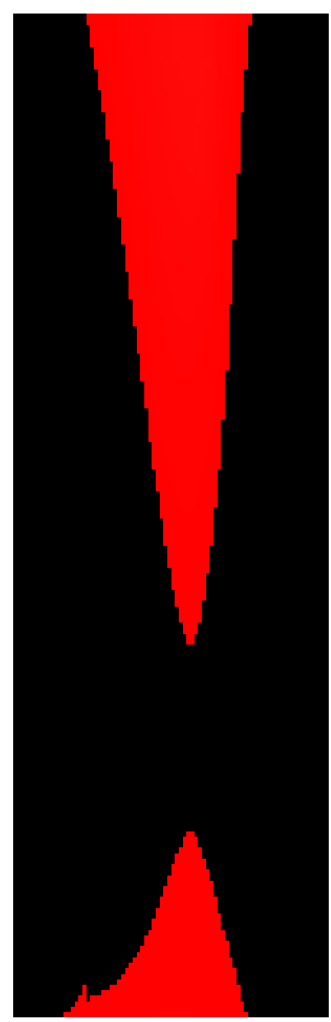

(a)

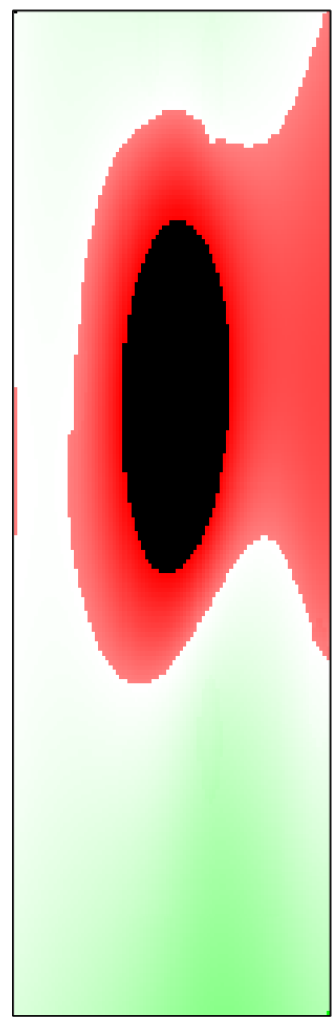

(b)

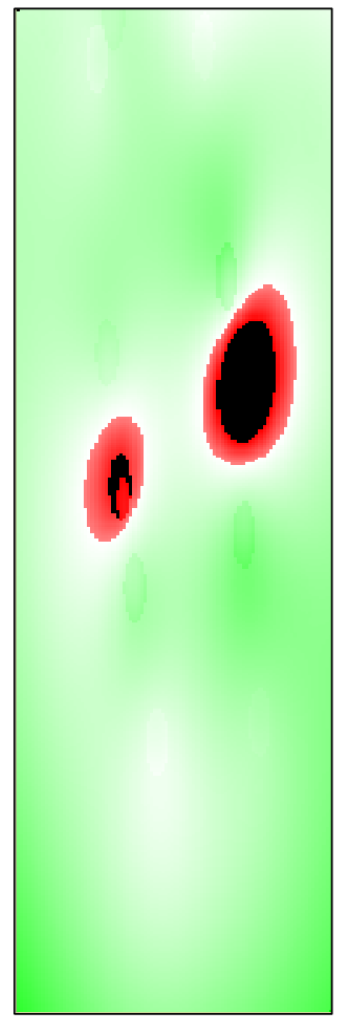

(c)

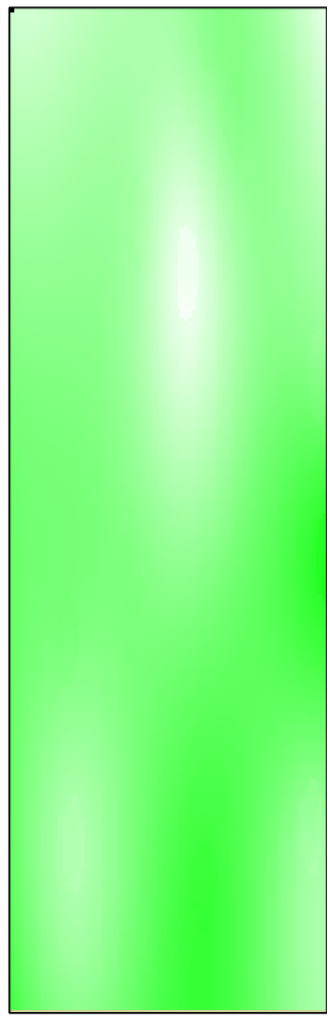

(d)

Fig. 10. Reendothelialization score maps for real acquisition data described in Tab. 0: (a) sequence \#0, (b) sequence \#1, (c) sequence \#3 and (d) sequence \#6.

$$
\begin{aligned}
\mathbf{w}_{m}^{(k)} & =\frac{\tilde{\mathbf{h}}_{m}^{\mathrm{T}} \mathbf{\Omega}^{(k)} \mathbf{r}_{m-1}}{\tilde{\mathbf{h}}_{m}^{\mathrm{T}} \boldsymbol{\Omega}^{(k)} \mathbf{h}_{m}} \\
\text { and } \mathbf{r}_{m}^{(k)} & =\mathbf{r}_{m-1}-\widetilde{\mathbf{w}}_{m}^{(k)} \tilde{\mathbf{h}}_{m},
\end{aligned}
$$

where the diagonal matrix $\boldsymbol{\Omega}^{(k)}$ is rebuilt at each iteration from the values of the first derivative of the Huber's function M-estimator $\rho$ at residuals $\mathbf{r}_{m}^{(k)}$ :

$\boldsymbol{\Omega}_{i, i}^{(k)}=\frac{\rho^{\prime}\left(\mathbf{r}_{m_{i}}^{(k)}\right)}{\mathbf{r}_{m_{i}}^{(k)}}=\left\{\begin{array}{c}1 \text { if }\left|\mathbf{r}_{m_{i}}^{(k)}\right| \leq 1.345 \operatorname{std}\left(\mathbf{r}_{m}^{(k)}\right) \\ 1.345 \operatorname{std}\left(\mathbf{r}_{m}^{(k)}\right) /\left|\mathbf{r}_{m_{i}}^{(k)}\right| \text { otherwise }\end{array}\right.$

where the standard deviation of residuals is robustly computed by the median absolute deviation. Robust regression will be referred to as RR in the following.

\section{RESULTS AND DISCUSSION}

\section{A. Evaluation of depth map interpolation}

To evaluate the benefits of the different stages of the map interpolation, they are evaluated on simulated data with irregularly-spaced points inferred from true locations of $p=$ 1600 struts (Fig. 10(a)). The depth function is arbitrarily chosen as the sum of a ramp over $\mathrm{z}$ of amplitude 255 and a sinusoidal function, also of amplitude 255, for both $\theta$ and $z$. In some simulation conditions, centered additive white Gaussian noise of standard deviation $\sigma$ is added to the theoretical model for the depth, and a given value is randomly added to or subtracted from the theoretical function for a given percentage $\varepsilon$ of the points to create outliers (Fig. 10(b)).

Figure 10(c) shows the result of the interpolation that is visually what is expected. Table 0 gives the mean errors to the ground truth on average for either the whole domain or for the struts only. It can be seen that, as expected, errors are globally lower at the struts compared to the whole domain, as the selection criterion is only based on measured values at the struts. Error on the domain obviously depends on the exact function profile and on the global density of measurement points, and is not generally correlated to the error at the struts.

However, the error values in table 0 show that every correction (DC, DA, GCV, RR) decreases the final error. Tests are performed without noise for DC and DA and without outliers (except for RR).

Simulations 1 to 4 prove that error is decreased by applying DA and DC alone, but the result is only slightly bettered when both corrections are cumulated. However, the difference lies in the distribution of errors, as precision is expected to be higher for small values.

Simulations 5 to 7 show the benefit of using the GCV criterion to select regularization parameter: for the same amount of additive Gaussian noise $\sigma=10$, simulation 5 gives an example of over-fitting $(\lambda=0)$, whereas simulation 6 corresponds to over-smoothing $(\lambda=0.1)$. Automatic selection gives a very low error value (simulation 7 ). The next simulation condition 8 show that changes in noise conditions $\sigma=5$ lead to a lower estimated regularization value $\lambda$.

Simulations 9 and 10 illustrate the influence of outlier level on the final result. Simulation 9 shows how both center selection and regularization estimation are sensitive to the presence of outliers. The proposed robust correction takes outliers into account, and the final error of simulation 10 is comparable to the value obtained without outliers ( 0.28 against 0.10). Figure 10 shows the difference (Fig. 10(e)) between the 
estimated distance map (Fig. 10(c)) and the expected map (Fig. a perfect cylindrical vessel bed. Shape irregularities almost TABLE I

Results of distance map interpolation for simulated data. Different simulation conditions are considered (Gaussian noise standard deviation $\sigma$ and percentage of outliers, $\varepsilon$ ). The regularization parameter $\lambda$ is an input except when the GCV criterion is used (simulations 7 to 10). The discrepancies between estimated and expected distances are given as averages for every strut location or globally for the whole map domain.

\begin{tabular}{|c|c|c|c|c|c|c|c|c|c|c|}
\hline Simulation \# & 1 & 2 & 3 & 4 & 5 & 6 & 7 & 8 & 9 & 10 \\
\hline Corrections & none & DA & DC & $D C+D A$ & $D C+D A$ & $D C+D A$ & $\mathrm{DC}+\mathrm{DA}+\mathrm{GCV}$ & $\mathrm{DC}+\mathrm{DA}+\mathrm{GCV}$ & $\mathrm{DC}+\mathrm{DA}+\mathrm{GCV}$ & $\mathrm{DC}+\mathrm{DA}+\mathrm{GCV}+\mathrm{RR}$ \\
\hline Conditions & $\sigma=0, \varepsilon=0$ & $\sigma=0, \varepsilon=0$ & $\sigma=0, \varepsilon=0$ & $\sigma=0, \varepsilon=0$ & $\sigma=10, \varepsilon=0$ & $\sigma=10, \varepsilon=0$ & $\sigma=10, \varepsilon=0$ & $\sigma=5, \varepsilon=0$ & $\sigma=10, \varepsilon=1$ & $\sigma=10, \varepsilon=1$ \\
\hline Parameters & $\lambda=1.0 \mathrm{e}-4$ & $\lambda=1.0 \mathrm{e}-4$ & $\lambda=1.0 \mathrm{e}-4$ & $\lambda=1.0 \mathrm{e}-4$ & $\lambda=0$ & $\lambda=0.1$ & $\lambda=7.6 \mathrm{e}-4$ & $\lambda=6.0 \mathrm{e}-4$ & $\lambda=4.3 \mathrm{e}-3$ & $\lambda=5.6 \mathrm{e}-3$ \\
\hline Mean error / strut & 2.65 & 0.87 & 0.52 & 0.50 & 0.51 & -0.95 & 0.10 & 0.079 & 0.91 & 0.28 \\
\hline Mean error / domain & 3.68 & 0.98 & -0.53 & -0.51 & -0.65 & 0.33 & -0.61 & -0.49 & -1.32 & -0.60 \\
\hline
\end{tabular}

TABLE II

Characteristics of the OCT sequences used for validation: percentage of shape irregularity alerts; detection rate is computed from the actual number of struts counted by the expert; and the number of struts used for comparisons between the supervised method and the gold standard (phantom) or manual measurements (vessel) is given.

\begin{tabular}{|c|c|c|c|c|c|c|c|c|c|c|c|c|}
\hline Sequence \# & 0 & 1 & 2 & 3 & 4 & 5 & 6 & 7 & 8 & 9 & 10 & 11 \\
\hline Number of frames & 183 & 210 & 412 & 396 & 346 & 403 & 282 & 242 & 406 & 196 & 317 & 195 \\
\hline Irregularity alerts (\%) & 0 & 4,3 & 19 & 7,3 & 0 & 0 & 1,1 & 2 & 0 & 0 & 5 & 0 \\
\hline Number of compared struts & 223 & 102 & 387 & 374 & 248 & 325 & 292 & 198 & 278 & 205 & 140 & 190 \\
\hline Strut detection rate (\%) & 84,44 & 35,42 & 73,39 & 62,24 & 54,84 & 36,15 & 34,30 & 42,17 & 41,65 & 36,59 & 53,57 & 49,47 \\
\hline
\end{tabular}

10(a)), in microns. Figure 10(b) gives the location of the struts used for interpolation: note that almost all theoretical outliers are rejected and only a few false detections occur in the corner of the plane $(\theta, z)$, where the model is less constrained by the data.

\section{B. Validation of reendothelialization measurement}

The parameters are analyzed in cross-sections taken every millimeter from the DICOM recordings obtained conformably to procedure described in paragraph II.A.1. Lumen Area (LA, in $\mathrm{mm}^{2}$ ) and Stent Area (SA, in $\mathrm{mm}^{2}$ ) were measured on each image to calculate the percentage of NeoIntimal Hyperplasia Area as NIHA $=100($ SA-LA $) /$ SA. Neointimal hyperplasia thickness was measured manually for each strut using the offline software delivered with the OCT console (LightLab Imaging, Westford, MA, USA).

For validation, 11 characteristic OCT exams were chosen, with coverage ranging from homogeneous low and high to inhomogeneous. Moreover a phantom acquisition consisting in a stent deployed into a $3 \mathrm{~mm}$ diameter urinary catheter (Fig. 10(a)) was used. The first row of Fig. 10 shows images with well-apposed stent (scores 0 or 1 ), whereas the second row gives different configurations that may occur over the length of a malapposition. The struts visible in each image were classified into 4 categories: (1) well-apposed to the vessel wall with apparent neointimal coverage (Fig. 10(b) and 10(c)); (2) well-apposed but without coverage (absence of any visible structure between lumen and vessel on OCT confirmed by a strong echo at the strut) (Fig. 10(a) and 10(c)), and (3) malapposed without coverage (malapposition being defined as a distance higher than the width of the strut, i.e. $150 \mu \mathrm{m}$ between the wall and the light echo) (Fig. 10(d), 10(e), 10(f)).

Table 0 summarizes the conditions of the comparison to the gold standard and to the manual measurement, performed on 3588 individual struts. The simple automatic quality control on vessel shape was applied to all the OCT sequences analyzed in this paper. Table 0 gives the number of alerts automatically detected for each OCT image sequence. The Alert Threshold $T_{A}$ value was set by the phantom analysis that can be considered as always occur at the endings of the malapposition area where the neointimal layer seems detached from the wall (Fig. 1(d) and 1(f)). But the expert intervention is not always necessary as deformable models can perform well with non concave shapes (Fig. 1(d)): on average, they only interact with 4 or $5 \%$ of the slices in malapposition cases.

The detection rate reported in Table 0 range from $35.42 \%$ to $73.39 \%$ in vivo and up to $84.44 \%$ in vitro. $35.42 \%$ could be considered as a poor detection rate, but in fact it corresponds to a mean density of 13 struts per square $\mathrm{mm}$, high enough to describe the whole depth surface, given it is smooth due to the stent stiffness and to the regularity of the neointimal coverage process.

Globally there is many fewer slices requiring expert evaluation than slices with coverage defects (score 1 or 2). For instance, for sequence \#1, there is a $4.3 \%$ alert rate for about $40 \%$ of uncovered struts.

Concerning area indices, estimation has a tendency to underestimate both lumen and stent areas. This is a systematic error that may be due to a default in distance scale in the console itself. The error is not that important since it is compensated in the final NIHA ratio (Fig. 10(a)), for which supervised estimation was in good agreement with manual measurement (bias equal to $-1.14 \%$ ). Both areas are found overestimated in the phantom case ( 7.72 against 7.07 for LA and 6.26 against 5.73 for SA) since gold standard values are computed from the nominal diameter of the urinary catheter. This does account for deformation caused by stent stiffness. However, again, area values are compensated in the ratio, giving very high agreement ( $0.23 \%$ in absolute difference).

For qualitative results (Fig. 10), distribution between score classes is comparable to a few percent-points between supervised and manual measurements (Fig. 10(b)). Score class 2 is sometimes underestimated to the benefit of score class 1 , but it is not serious because both require the expert cardiologist to be warned. Again, the main discrepancy between measurement and expectation occurs for the phantom, but this is explained simply by the fact that the distance measured 
between the wall and the light echo is at a few microns higher or lower than the limit between score 1 and 2, i.e. $150 \mu \mathrm{m}$. Therefore, the score map oscillates between red and black (Fig. $10(a))$.

Concerning quantitative results, Fig. 10(c) shows that there is almost no bias between the two methods: supervised method over-estimates at about $5 \mu \mathrm{m}$, i.e. less than $20 \mu \mathrm{m}$, which is the resolution of OCT. It is interesting to note that standard deviation depends mostly on the magnitude of the coverage, being higher for sequence \#6 for instance $(\mathrm{SD}=109.5 \mu \mathrm{m})$ which has higher coverage than sequence \#1 ( $\mathrm{SD}=49.99 \mu \mathrm{m})$ with similar detection rates. Furthermore, standard deviation predictably increases with coverage inhomogeneity: SD is of the same order of magnitude for homogenous sequence \#6 and inhomogeneous sequence \#3, whereas double the number of struts are detected in the latter.

For now, the segmentation software prototype has been written in interface description language (IDL), so execution time is not yet optimized. Segmentation tasks were performed on a Pentium $43.4 \mathrm{GHz}$ CPU with $1 \mathrm{~GB}$ of RAM. Computation time was $0.8 \mathrm{~s}$ per slice for lumen and strut detection. If user interaction is required, semi-automatic correction takes about 5 to 20 seconds per slice (the user initializes the contour that converges to the boundary as an Active Model). This means that the whole segmentation process may take up to one quarterhour for highly irregular OCT sequences, compared to the full manual process that takes the same time for only one slice. This duration must be compared to the full manual expert segmentation that lasts several hours per OCT sequence.

The score map prototype has been developed in $\mathrm{C}++$ and runs on an architecture similar to the previous one. Computation of a map from distances measured at $p=1600$ struts takes less than 2 minutes.

\section{CONCLUSION}

We have developed a processing chain that gives an accurate and robust continuous map of distance between the stent and the vessel wall. It has been converted into a color score for visualization convenience. Extra work is in progress to design a friendly real-time interface that enables to locate suspected zones of non coverage or malapposition in the initial OCT data and in $3 \mathrm{D}$ on the exact segment geometry. Ameliorations in vessel wall detection are also required in order to minimize user interaction.

\section{REFERENCES}

[1] A.V. Finn, M. Joner, G. Nakazawa, F.D. Kolodgie, J. Newell, M.C. John, H.K. Gold, and R. Virmani "Pathological correlates of late drug-eluting stent thrombosis: strut coverage as a marker of endothelialization," Circulation, vol. 115, no. 18, pp. 2435-2441, 2007.

[2] A.V. Finn, G. Nakazawa, M. Joner, F.D. Kolodgie, E.K. Mont, H.K. Gold, and R. Virmani, "Vascular responses to drug eluting stents: importance of delayed healing," Arterioscler. Thromb. Vasc. Biol., vol. 27, no. 7, pp. 1500-1510, 2007.

[3] T.L. Pinto and R. Waksman, "Clinical applications of optical coherence tomography," J. Interv. Cardiol., vol. 19, no. 6, pp. 566-573, 2006.

[4] Z.H. Yao, T. Matsubara, T. Inada, Y. Suzuki, and T. Suzuki, "Neointimal coverage of sirolimus-eluting stents 6 months and 12 months after implantation: evaluation by optical coherence tomography," Chin. Med. J., vol. 121, no. 6, pp. 503-507, 2008.

[5] B.X. Chen, F.Y. Ma, W. Luo, J.H. Ruan, W.L. Xie, X.Z. Zhao, S.H. Sun, X.M. Guo, F. Wang, T. Tian, and X.W. Chu "Neointimal coverage of bare-metal and sirolimus-eluting stents evaluated with optical coherence tomography," Heart, vol. 94, no. 5, pp. 566-570, 2008.

[6] D. Matsumoto, J. Shite, T. Shinke, H. Otake, Y. Tanino, D. Ogasawara, T. Sawada, O.L. Paredes, K. H., and M. Yokoyama, "Neointimal coverage of sirolimus-eluting stents at 6-month follow-up: evaluated by optical coherence tomography," Eur. Heart J., vol. 28, no. 8, pp. 961-967, 2007.

[7] G.T. Bonnema, K.O. Cardinal, J.B. McNally, S.K. Williams, and J.K. Barton, "Assessment of blood vessel mimics with optical coherence tomography," J. Biomed. Opt., vol. 12, no. 2, pp. 024018, 2007.

[8] S. Tanimoto, G. Rodriguez-Granillo, P. Barlis, S. de Winter, N. Bruining, R. Hamers, M. Knappen, S. Verheye, P. Serruys, and E. Regar, "A novel approach for quantitative analysis of intracoronary optical coherence tomography: high inter-observer agreement with computer-assisted contour detection," Catheter Cardiovasc. Interv., vol. 72, no. 2, pp. 228235, 2008.

[9] M.E. Brezinski, "Optical coherence tomography: principles and applications," Burlington, MA: Elsevier, 2006.

[10] G. Finet, C. Cachard, P. Delachartre, E. Maurincomme, and J. Beaune, "Artifacts in intravascular ultrasound imaging during coronary artery stent implantation," Ultrasound Med. Biol., vol. 14, no. 6, pp. 793-802, 1998.

[11] C. Kauffmann, B. Godbout, and J. A. de Guise, "Simplified active contour model applied to bone structure segmentation in digital radiographs," in Proc. SPIE Medical Imaging, pp. 663-672, 1998.

[12] N. Otsu, "A threshold selection method from gray-scale histogram," IEEE Trans. Systems, Man, and Cybernetics, vol. 8, pp. 62-66, 1978

[13] A. Fitzgibbon, M. Pilu, and R. B. Fisher, "Direct least square fitting of ellipses," IEEE Trans. Pattern Analysis and Machine Intelligence, pp. 476-480, 1999.

[14] G. Donato and S. Belongie, "Approximate thin plate spline mappings," Lecture Notes in Computer Science, Vol. 2352, Proc. European Conference on Computer Vision-Part III, pp. 21-31, 2002.

[15] S. Chen, C.F.N. Cowan, and P.M. Grant, "Orthogonal least squares learning for radial basis function network," IEEE Trans. Neural Networks, vol. 2, no. 2, pp. 302-309, 1991.

[16] F.L. Bookstein, "Principal warps: thin-plate splines and the decomposition of deformations," IEEE Trans. Pattern Anal. Mach. Intell., vol. 11 , no. 6, 1989.

[17] M.J.L. Orr, "Regularisation in the selection of radial basis function centres," Neural Computation, vol. 7, no. 3, pp. 606-623, 1995.

[18] G. Wahba, "Spline Models for Observational Data," CBSM-NSF Regional Conf. Ser. Appl. Math. 59, Philadelphia: SIAM, 1990.

[19] A. Bartoli, "Maximizing the predictivity of smooth deformable image warps through cross-validation," J. Math. Imaging Vis., vol. 31, no. 2-3, pp. 133-145, 2008.

[20] X. Hong and S. Chen, "M-estimator and D-optimality model construction using orthogonal forward regression," IEEE Trans. System, Man, and Cybernetics - Part B, vol. 35, no. 1, pp. 155-162, 2005.

$1,2,3,4,5,6,7,8,9,10$

1,2 